\title{
Proof of two divisibility properties of binomial coefficients conjectured by Z.-W. Sun
}

\author{
Victor J. W. Guo \\ Department of Mathematics, Shanghai Key Laboratory of PMMP, \\ East China Normal University, 500 Dongchuan Road, \\ Shanghai 200241, People's Republic of China \\ jwguo@math.ecnu.edu.cn, http://math.ecnu.edu.cn/ ${ }^{j}$ wguo
}

Submitted: May 4, 2014; Accepted: Jun 8, 2014; Published: Jun 27, 2014

Mathematics Subject Classifications: 11B65, 05A10, 05A30

\begin{abstract}
For all positive integers $n$, we prove the following divisibility properties:

$$
(2 n+3)\left(\begin{array}{c}
2 n \\
n
\end{array}\right) \mid 3\left(\begin{array}{c}
6 n \\
3 n
\end{array}\right)\left(\begin{array}{c}
3 n \\
n
\end{array}\right) \quad \text { and } \quad(10 n+3)\left(\begin{array}{c}
3 n \\
n
\end{array}\right) \mid 21\left(\begin{array}{c}
15 n \\
5 n
\end{array}\right)\left(\begin{array}{c}
5 n \\
n
\end{array}\right)
$$

This confirms two recent conjectures of Z.-W. Sun. Some similar divisibility properties are given. Moreover, we show that, for all positive integers $m$ and $n$, the product $a m\left(\begin{array}{c}a m+b m-1 \\ a m\end{array}\right)\left(\begin{array}{c}a n+b n \\ a n\end{array}\right)$ is divisible by $m+n$. In fact, the latter result can be further generalized to the $q$-binomial coefficients and $q$-integers case, which generalizes the positivity of $q$-Catalan numbers. We also propose several related conjectures.
\end{abstract}

Keywords: congruences, binomial coefficients, $p$-adic order, $q$-Catalan numbers, reciprocal and unimodal polynomials

\section{Introduction}

In $[18,19]$, Z.-W. Sun proved some divisibility properties of binomial coefficients, such as

$$
\begin{aligned}
& 2(2 n+1)\left(\begin{array}{c}
2 n \\
n
\end{array}\right) \mid\left(\begin{array}{c}
6 n \\
3 n
\end{array}\right)\left(\begin{array}{c}
3 n \\
n
\end{array}\right), \\
& (10 n+1)\left(\begin{array}{c}
3 n \\
n
\end{array}\right) \mid\left(\begin{array}{c}
15 n \\
5 n
\end{array}\right)\left(\begin{array}{c}
5 n-1 \\
n-1
\end{array}\right) .
\end{aligned}
$$


Some similar divisibility results were later obtained by Guo [10] and Guo and Krattenthaler [11]. A generalization of (1.1) was recently given by Sepanski [15]. It is worth mentioning that Bober [6] has completely described when ratios of factorial products of the form

$$
\frac{\left(a_{1} n\right) ! \cdots\left(a_{k} n\right) !}{\left(b_{1} n\right) ! \cdots\left(b_{k+1} n\right) !}
$$

with $a_{1}+\cdots+a_{k}=b_{1}+\cdots+b_{k+1}$ are always integers.

Let

$$
S_{n}=\frac{\left(\begin{array}{c}
6 n \\
3 n
\end{array}\right)\left(\begin{array}{c}
3 n \\
n
\end{array}\right)}{2(2 n+1)\left(\begin{array}{c}
2 n \\
n
\end{array}\right)}, \quad \text { and } \quad t_{n}=\frac{\left(\begin{array}{c}
15 n \\
5 n
\end{array}\right)\left(\begin{array}{c}
5 n-1 \\
n-1
\end{array}\right)}{(10 n+1)\left(\begin{array}{c}
3 n \\
n
\end{array}\right)}
$$

In this paper we first prove the following two results conjectured by Z.-W. Sun [18, 19].

Theorem 1.1 (see [18, Conjecture 3(i)]) Let $n$ be a positive integer. Then

$$
3 S_{n} \equiv 0 \quad(\bmod 2 n+3) .
$$

Theorem 1.2 [19, Conjecture 1.3] Let $n$ be a positive integer. Then

$$
21 t_{n} \equiv 0 \quad(\bmod 10 n+3) .
$$

We shall also give more congruences for $S_{n}$ and $t_{n}$ as follows.

Theorem 1.3 Let $n$ be a positive integer. Then

$$
\begin{aligned}
& 105 S_{n} \equiv 0 \quad(\bmod 2 n+5), \\
& 315 S_{n} \equiv 0 \quad(\bmod 2 n+7), \\
& 6435 S_{n} \equiv 0 \quad(\bmod 2 n+9), \\
& 3003 t_{n} \equiv 0 \quad(\bmod 2 n+1), \\
& 88179 t_{n} \equiv 0 \quad(\bmod 10 n+7), \\
& 43263 t_{n} \equiv 0 \quad(\bmod 10 n+9) .
\end{aligned}
$$

Let $\mathbb{Z}$ denote the set of integers. Another result in this paper is the following.

Theorem 1.4 Let $a, b, m, n$ be positive integers. Then

$$
\frac{a b m}{(a+b)(m+n)}\left(\begin{array}{c}
a m+b m \\
a m
\end{array}\right)\left(\begin{array}{c}
a n+b n \\
a n
\end{array}\right)=\frac{a m}{m+n}\left(\begin{array}{c}
a m+b m-1 \\
a m
\end{array}\right)\left(\begin{array}{c}
a n+b n \\
a n
\end{array}\right) \in \mathbb{Z} .
$$

Letting $a=b=1$ in (1.10), we get the following result, of which a combinatorial interpretation was given by Gessel [9, Section 7].

Corollary 1.5 Let $m, n$ be positive integers. Then

$$
\frac{m}{2(m+n)}\left(\begin{array}{c}
2 m \\
m
\end{array}\right)\left(\begin{array}{c}
2 n \\
n
\end{array}\right) \in \mathbb{Z} \text {. }
$$

In the next section, we give some lemmas. The proofs of Theorems 1.1-1.3 will be given in Sections 3-5 respectively. A proof of the $q$-analogue of Theorem 1.4 will be given in Section 6. We close our paper with some further remarks and open problems in Section 7. 


\section{Some lemmas}

For the $p$-adic order of $n$ !, there is a known formula

$$
\operatorname{ord}_{p} n !=\sum_{i=1}^{\infty}\left\lfloor\frac{n}{p^{i}}\right\rfloor,
$$

where $\lfloor x\rfloor$ denotes the greatest integer not exceeding $x$. In this section, we give some results on the floor function $\lfloor x\rfloor$.

Lemma 2.1 For any real number $x$, we have

$$
\begin{aligned}
\lfloor 6 x\rfloor+\lfloor x\rfloor & \geqslant\lfloor 3 x\rfloor+2\lfloor 2 x\rfloor, \\
\lfloor 15 x\rfloor+\lfloor 2 x\rfloor & \geqslant\lfloor 10 x\rfloor+\lfloor 4 x\rfloor+\lfloor 3 x\rfloor .
\end{aligned}
$$

Proof. See [6, Theorem 1.1] and one of the 52 sporadic step functions given in [6, Table 2, line\# 32].

Lemma 2.2 Let $m$ and $n$ be two positive integers such that $m \mid 2 n+3$ and $m \geqslant 5$. Then

$$
\left\lfloor\frac{6 n}{m}\right\rfloor+\left\lfloor\frac{n}{m}\right\rfloor=\left\lfloor\frac{3 n}{m}\right\rfloor+2\left\lfloor\frac{2 n}{m}\right\rfloor+1 .
$$

Proof. Let $\{x\}=x-\lfloor x\rfloor$ be the fractional part of $x$. Then (2.4) is equivalent to

$$
\left\{\frac{6 n}{m}\right\}+\left\{\frac{n}{m}\right\}=\left\{\frac{3 n}{m}\right\}+2\left\{\frac{2 n}{m}\right\}-1 .
$$

Now suppose that $m \mid 2 n+3$ and $m \geqslant 5$. We have

$$
\left\{\frac{2 n}{m}\right\}=\frac{m-3}{m}>\frac{1}{3}, \quad \text { and } \quad\left\lfloor\frac{2 n}{m}\right\rfloor=\frac{2 n+3}{m}-1 \equiv 0 \quad(\bmod 2) .
$$

It follows that

$$
\begin{gathered}
\left\{\frac{6 n}{m}\right\}= \begin{cases}\frac{2 m-9}{m}, & \text { if } m=5,7, \\
\frac{m-9}{m}, & \text { if } m \geqslant 9,\end{cases} \\
\left\{\frac{n}{m}\right\}=\frac{m-3}{2 m}, \\
\left\{\frac{3 n}{m}\right\}= \begin{cases}\frac{3 m-9}{2 m}, & \text { if } m=5,7, \\
\frac{m-9}{2 m}, & \text { if } m \geqslant 9 .\end{cases}
\end{gathered}
$$

Therefore, the identity $(2.5)$ is true for any positive integer $m \geqslant 5$. 
Lemma 2.3 Let $m$ and $n$ be two positive integers such that $m \mid 10 n+3$ and $m \geqslant 9$. Then

$$
\left\lfloor\frac{15 n}{m}\right\rfloor+\left\lfloor\frac{2 n}{m}\right\rfloor=\left\lfloor\frac{10 n}{m}\right\rfloor+\left\lfloor\frac{4 n}{m}\right\rfloor+\left\lfloor\frac{3 n}{m}\right\rfloor+1 .
$$

Proof. It is easy to see that (2.6) is equivalent to

$$
\left\{\frac{15 n}{m}\right\}+\left\{\frac{2 n}{m}\right\}=\left\{\frac{10 n}{m}\right\}+\left\{\frac{4 n}{m}\right\}+\left\{\frac{3 n}{m}\right\}-1
$$

Now suppose that $m \mid 10 n+3$ and $m \geqslant 9$. We have

$$
\left\{\frac{10 n}{m}\right\}=\frac{m-3}{m} \geqslant \frac{2}{3}, \quad \text { and } \quad A:=\left\lfloor\frac{10 n}{m}\right\rfloor=\frac{10 n+3}{m}-1 \equiv 0,2,6,8 \quad(\bmod 10) .
$$

It is easy to check that

$$
\begin{gathered}
\left\{\frac{15 n}{m}\right\}=\frac{m-9}{2 m}, \\
\left(\left\{\frac{2 n}{m}\right\},\left\{\frac{4 n}{m}\right\},\left\{\frac{3 n}{m}\right\}\right)= \begin{cases}\left(\frac{2 m-6}{10 m}, \frac{4 m-12}{10 m}, \frac{3 m-9}{10 m}\right), & \text { if } A \equiv 0 \quad(\bmod 10), \\
\left(\frac{6 m-6}{10 m}, \frac{2 m-12}{10 m}, \frac{9 m-9}{10 m}\right), & \text { if } A \equiv 2 \quad(\bmod 10), \\
\left(\frac{4 m-6}{10 m}, \frac{8 m-12}{10 m}, \frac{m-9}{10 m}\right), & \text { if } A \equiv 6 \quad(\bmod 10), \\
\left(\frac{8 m-6}{10 m}, \frac{6 m-12}{10 m}, \frac{7 m-9}{10 m}\right), & \text { if } A \equiv 8 \quad(\bmod 10),\end{cases}
\end{gathered}
$$

and so the identity (2.7) holds.

\section{Proofs of Theorem 1.1}

First Proof. Let $\operatorname{gcd}(a, b)$ denote the greatest common divisor of two integers $a$ and $b$. For any positive integer $n$, since $\operatorname{gcd}(2 n+3,4 n+2)=1$, to prove Theorem 1.1 , it is enough to show that

$$
(2 n+3) \mid \frac{3\left(\begin{array}{c}
6 n \\
3 n
\end{array}\right)\left(\begin{array}{c}
3 n \\
n
\end{array}\right)}{\left(\begin{array}{c}
2 n \\
n
\end{array}\right)}
$$

By (2.1), for any odd prime $p$, the $p$-adic order of

$$
\frac{\left(\begin{array}{c}
6 n \\
3 n
\end{array}\right)\left(\begin{array}{c}
3 n \\
n
\end{array}\right)}{(2 n+3)\left(\begin{array}{c}
2 n \\
n
\end{array}\right)}=\frac{(2 n+2) !(6 n) !(n) !}{(2 n+3) !(3 n) !(2 n) !^{2}}
$$


is given by

$$
\sum_{i=1}^{\infty}\left(\left\lfloor\frac{2 n+2}{p^{i}}\right\rfloor+\left\lfloor\frac{6 n}{p^{i}}\right\rfloor+\left\lfloor\frac{n}{p^{i}}\right\rfloor-\left\lfloor\frac{2 n+3}{p^{i}}\right\rfloor-\left\lfloor\frac{3 n}{p^{i}}\right\rfloor-2\left\lfloor\frac{2 n}{p^{i}}\right\rfloor\right) .
$$

Note that

$$
\left\lfloor\frac{2 n+2}{p^{i}}\right\rfloor-\left\lfloor\frac{2 n+3}{p^{i}}\right\rfloor= \begin{cases}-1, & \text { if } p^{i} \mid 2 n+3 \\ 0, & \text { otherwise. }\end{cases}
$$

By Lemmas 2.1 and 2.2, for $p \geqslant 5$, the summation (3.2) is clearly greater than or equal to 0 . For $p=3$, we have $(3.2) \geqslant-1$ because if the positive integer $i$ satisfies $3^{i} \mid 2 n+3$ and $3^{i}<5$ then we must have $i=1$. This proves that

$$
\frac{3\left(\begin{array}{c}
6 n \\
3 n
\end{array}\right)\left(\begin{array}{c}
3 n \\
n
\end{array}\right)}{(2 n+3)\left(\begin{array}{c}
2 n \\
n
\end{array}\right)}
$$

is always an integer. Hence (3.1) holds.

Second Proof (provided by T. Amdeberhan and V.H. Moll). Replacing $n$ by $n+1$ in (1.1), we see that (after some rearrangement)

$$
\frac{\left(\begin{array}{c}
6 n+6 \\
3 n+3
\end{array}\right)\left(\begin{array}{c}
3 n+3 \\
n+1
\end{array}\right)}{2(2 n+3)\left(\begin{array}{c}
2 n+2 \\
n+1
\end{array}\right)}=\frac{6(6 n+5)(6 n+1) S_{n}}{(n+1)(2 n+3)} \in \mathbb{Z} .
$$

Hence, $(2 n+3) \mid 6(6 n+5)(6 n+1) S_{n}$. Since $\operatorname{gcd}(2 n+3,2)=\operatorname{gcd}(2 n+3,6 n+5)=$ $\operatorname{gcd}(2 n+3,6 n+1)=1$, we must have $(2 n+3) \mid 3 S_{n}$.

Remark. Z.-W. Sun [18, Conjecture 3(i)] also conjectured that $S_{n}$ is odd if and only if $n$ is a power of 2. After reading a previous version of this paper, Quan-Hui Yang told me that it is easy to show that $\operatorname{ord}_{2}\left((6 n) ! n ! /(3 n) !(2 n) !^{2}\right)$ equals the number of 1 's in the binary expansion of $n$ by noticing

$$
\operatorname{ord}_{2}(6 n) !=3 n+\operatorname{ord}_{2}(3 n) !, \quad \operatorname{ord}_{2}(2 n) !=n+\operatorname{ord}_{2} n !,
$$

and using Legendre's theorem. T. Amdeberhan and V.H. Moll also pointed out this.

\section{Proof of Theorem 1.2}

For any positive integer $n$, since $\operatorname{gcd}(10 n+3,10 n+1)=1$, to prove Theorem 1.2 , it is enough to show that

$$
(10 n+3) \mid \frac{21\left(\begin{array}{c}
15 n \\
5 n
\end{array}\right)\left(\begin{array}{c}
5 n-1 \\
n-1
\end{array}\right)}{\left(\begin{array}{c}
3 n \\
n
\end{array}\right)}
$$


Furthermore, since $\operatorname{gcd}(10 n+3,5)=1$ and $\left(\begin{array}{c}5 n \\ n\end{array}\right)=5\left(\begin{array}{c}5 n-1 \\ n-1\end{array}\right)$, one sees that (4.1) is equivalent to

$$
(10 n+3) \mid \frac{21\left(\begin{array}{c}
15 n \\
5 n
\end{array}\right)\left(\begin{array}{c}
5 n \\
n
\end{array}\right)}{\left(\begin{array}{c}
3 n \\
n
\end{array}\right)}
$$

By (2.1), for any odd prime $p$, the $p$-adic order of

$$
\frac{\left(\begin{array}{c}
15 n \\
5 n
\end{array}\right)\left(\begin{array}{c}
5 n \\
n
\end{array}\right)}{(10 n+3)\left(\begin{array}{c}
3 n \\
n
\end{array}\right)}=\frac{(10 n+2) !(15 n) !(2 n) !}{(10 n+3) !(10 n) !(4 n) !(3 n) !}
$$

is given by

$$
\sum_{i=1}^{\infty}\left(\left\lfloor\frac{10 n+2}{p^{i}}\right\rfloor+\left\lfloor\frac{15 n}{p^{i}}\right\rfloor+\left\lfloor\frac{2 n}{p^{i}}\right\rfloor-\left\lfloor\frac{10 n+3}{p^{i}}\right\rfloor-\left\lfloor\frac{10 n}{p^{i}}\right\rfloor-\left\lfloor\frac{4 n}{p^{i}}\right\rfloor-\left\lfloor\frac{3 n}{p^{i}}\right\rfloor\right) .
$$

Note that

$$
\left\lfloor\frac{10 n+2}{p^{i}}\right\rfloor-\left\lfloor\frac{10 n+3}{p^{i}}\right\rfloor= \begin{cases}-1, & \text { if } p^{i} \mid 10 n+3 \\ 0, & \text { otherwise. }\end{cases}
$$

By Lemmas 2.1 and 2.3, for $p \geqslant 11$, or $p=5$, the summation (4.3) is clearly greater than or equal to 0 . For $p=3,7$, we have $(4.3) \geqslant-1$ because there is at most one index $i \geqslant 1$ satisfying $p^{i} \mid 10 n+3$ and $p^{i}<9$ in this case. This proves that

$$
\frac{21\left(\begin{array}{c}
15 n \\
5 n
\end{array}\right)\left(\begin{array}{c}
5 n \\
n
\end{array}\right)}{(10 n+3)\left(\begin{array}{c}
3 n \\
n
\end{array}\right)}
$$

is always an integer. Namely, (4.2) is true.

\section{$5 \quad$ Proof of Theorem 1.3}

Lemma 5.1 Let $m$ and $n$ be two positive integers. Then

$$
\left\lfloor\frac{6 n}{m}\right\rfloor+\left\lfloor\frac{n}{m}\right\rfloor=\left\lfloor\frac{3 n}{m}\right\rfloor+2\left\lfloor\frac{2 n}{m}\right\rfloor+1
$$

if $m \mid 2 n+5$ and $m \geqslant 9$, or $m \mid 2 n+7$ and $m \geqslant 11$, or $m \mid 2 n+9$ and $m \geqslant 15$.

Proof. The proof is similar to that of Lemma 2.2. We only consider the case when $m \mid 2 n+5$ and $m \geqslant 9$. In this case, we have

$$
\left\{\frac{2 n}{m}\right\}=\frac{m-5}{m}>\frac{1}{3}, \quad \text { and } \quad\left\lfloor\frac{2 n}{m}\right\rfloor=\frac{2 n+5}{m}-1 \equiv 0 \quad(\bmod 2) .
$$


It follows that

$$
\begin{aligned}
& \left\{\frac{6 n}{m}\right\}= \begin{cases}\frac{2 m-15}{m}, & \text { if } m=9,11,13 \\
\frac{m-15}{m}, & \text { if } m \geqslant 15,\end{cases} \\
& \left\{\frac{n}{m}\right\}=\frac{m-5}{2 m}, \\
& \left\{\frac{3 n}{m}\right\}= \begin{cases}\frac{3 m-15}{2 m}, & \text { if } m=9,11,13 \\
\frac{m-15}{2 m}, & \text { if } m \geqslant 15\end{cases}
\end{aligned}
$$

and so

$$
\left\{\frac{6 n}{m}\right\}+\left\{\frac{n}{m}\right\}=\left\{\frac{3 n}{m}\right\}+2\left\{\frac{2 n}{m}\right\}-1 .
$$

This proves (5.1).

Lemma 5.2 Let $m$ and $n$ be two positive integers. Then

$$
\left\lfloor\frac{15 n}{m}\right\rfloor+\left\lfloor\frac{2 n}{m}\right\rfloor=\left\lfloor\frac{10 n}{m}\right\rfloor+\left\lfloor\frac{4 n}{m}\right\rfloor+\left\lfloor\frac{3 n}{m}\right\rfloor+1
$$

if $m \mid 2 n+1$ and $m \geqslant 15$, or $m \mid 10 n+7$ and $m \geqslant 21$, or $m \mid 10 n+9$ and $m \geqslant 27$.

Proof. The proof is similar to that of Lemma 2.3. We only consider the case when $m \mid 10 n+9$ and $m \geqslant 27$. In this case, we have

$$
\left\{\frac{10 n}{m}\right\}=\frac{m-9}{m} \geqslant \frac{2}{3}, \quad \text { and } \quad A:=\left\lfloor\frac{10 n}{m}\right\rfloor=\frac{10 n+9}{m}-1 \equiv 0,2,6,8 \quad(\bmod 10) .
$$

It follows that

$$
\begin{gathered}
\left\{\frac{15 n}{m}\right\}=\frac{m-27}{2 m}, \\
\left(\left\{\frac{2 n}{m}\right\},\left\{\frac{4 n}{m}\right\},\left\{\frac{3 n}{m}\right\}\right)=\left\{\begin{array}{lll}
\left(\frac{2 m-18}{10 m}, \frac{4 m-36}{10 m}, \frac{3 m-27}{10 m}\right), & \text { if } A \equiv 0 \quad(\bmod 10), \\
\left(\frac{6 m-18}{10 m}, \frac{2 m-36}{10 m}, \frac{9 m-27}{10 m}\right), & \text { if } A \equiv 2 \quad(\bmod 10), \\
\left(\frac{4 m-18}{10 m}, \frac{8 m-36}{10 m}, \frac{m-27}{10 m}\right), & \text { if } A \equiv 6 \quad(\bmod 10), \\
\left(\frac{8 m-18}{10 m}, \frac{6 m-36}{10 m}, \frac{7 m-27}{10 m}\right), & \text { if } A \equiv 8 \quad(\bmod 10) .
\end{array}\right.
\end{gathered}
$$


Hence,

$$
\left\{\frac{15 n}{m}\right\}+\left\{\frac{2 n}{m}\right\}=\left\{\frac{10 n}{m}\right\}+\left\{\frac{4 n}{m}\right\}+\left\{\frac{3 n}{m}\right\}-1
$$

which means that (5.2) holds.

Proof of Theorem 1.3. Since the proofs of the congruences (1.4)-(1.9) are similar in view of Lemmas 5.1 and 5.2, we only give proofs of (1.5) and (1.9). Noticing that $\operatorname{gcd}(2 n+$ $1,2 n+7)=1$ or 3 , to prove (1.5), it suffices to show that

$$
(2 n+7) \mid \frac{105\left(\begin{array}{c}
6 n \\
3 n
\end{array}\right)\left(\begin{array}{c}
3 n \\
n
\end{array}\right)}{\left(\begin{array}{c}
2 n \\
n
\end{array}\right)} .
$$

Let

$$
X_{n}:=\frac{\left(\begin{array}{c}
6 n \\
3 n
\end{array}\right)\left(\begin{array}{c}
3 n \\
n
\end{array}\right)}{(2 n+7)\left(\begin{array}{c}
2 n \\
n
\end{array}\right)}=\frac{(2 n+6) !(6 n) !(n) !}{(2 n+7) !(3 n) !(2 n) !^{2}} .
$$

By (2.1), for any odd prime $p$, we have

$$
\operatorname{ord}_{p} X_{n}=\sum_{i=1}^{\infty}\left(\left\lfloor\frac{2 n+6}{p^{i}}\right\rfloor+\left\lfloor\frac{6 n}{p^{i}}\right\rfloor+\left\lfloor\frac{n}{p^{i}}\right\rfloor-\left\lfloor\frac{2 n+7}{p^{i}}\right\rfloor-\left\lfloor\frac{3 n}{p^{i}}\right\rfloor-2\left\lfloor\frac{2 n}{p^{i}}\right\rfloor\right) .
$$

Note that $(5.1)$ is also true for $m=3$ and $n \equiv 1(\bmod 3)$, and

$$
\left\lfloor\frac{2 n+6}{p^{i}}\right\rfloor-\left\lfloor\frac{2 n+7}{p^{i}}\right\rfloor= \begin{cases}-1, & \text { if } p^{i} \mid 2 n+7 \\ 0, & \text { otherwise }\end{cases}
$$

By Lemmas 2.1 and 5.1, we obtain

$$
\begin{cases}\operatorname{ord}_{p} X_{n} \geqslant 0, & \text { if } p \geqslant 11 \\ \operatorname{ord}_{p} X_{n} \geqslant-1, & \text { if } p=3,5,7\end{cases}
$$

This proves (5.3).

Similarly, since $\operatorname{gcd}(10 n+9,10 n+1)=\operatorname{gcd}(10 n+9,5)=1$, the congruence $(1.9)$ is equivalent to

$$
(10 n+9) \mid \frac{43263\left(\begin{array}{c}
15 n \\
5 n
\end{array}\right)\left(\begin{array}{c}
5 n \\
n
\end{array}\right)}{\left(\begin{array}{c}
3 n \\
n
\end{array}\right)} .
$$

Let

$$
Y_{n}:=\frac{\left(\begin{array}{c}
15 n \\
5 n
\end{array}\right)\left(\begin{array}{c}
5 n \\
n
\end{array}\right)}{(10 n+9)\left(\begin{array}{c}
3 n \\
n
\end{array}\right)}=\frac{(10 n+8) !(15 n) !(2 n) !}{(10 n+9) !(10 n) !(4 n) !(3 n) !}
$$


Then, for any odd prime $p, \operatorname{ord}_{p} Y_{n}$ is given by

$$
\sum_{i=1}^{\infty}\left(\left\lfloor\frac{10 n+8}{p^{i}}\right\rfloor+\left\lfloor\frac{15 n}{p^{i}}\right\rfloor+\left\lfloor\frac{2 n}{p^{i}}\right\rfloor-\left\lfloor\frac{10 n+9}{p^{i}}\right\rfloor-\left\lfloor\frac{10 n}{p^{i}}\right\rfloor-\left\lfloor\frac{4 n}{p^{i}}\right\rfloor-\left\lfloor\frac{3 n}{p^{i}}\right\rfloor\right) .
$$

Note that (5.2) also holds for $m=7,13,17$ and any positive integer $n$ such that $m \mid 10 n+9$. Similarly as before, we have

$$
\begin{cases}\operatorname{ord}_{p} Y_{n} \geqslant 0, & \text { if } p=5,7,13,17, \text { or } p \geqslant 29 \\ \operatorname{ord}_{p} Y_{n} \geqslant-1, & \text { if } p=11,19,23, \\ \operatorname{ord}_{p} Y_{n} \geqslant-2, & \text { if } p=3 .\end{cases}
$$

Observing that $43263=3^{2} \cdot 11 \cdot 19 \cdot 23$, we complete the proof of $(5.4)$.

\section{A $q$-analogue of Theorem 1.4}

Recall that the q-binomial coefficients are defined by

$$
\left[\begin{array}{l}
n \\
k
\end{array}\right]_{q}= \begin{cases}\frac{\left(1-q^{n}\right)\left(1-q^{n-1}\right) \cdots\left(1-q^{n-k+1}\right)}{(1-q)\left(1-q^{2}\right) \cdots\left(1-q^{k}\right)}, & \text { if } 0 \leqslant k \leqslant n \\
0, & \text { otherwise }\end{cases}
$$

We begin with the announced strengthening of Theorem 1.4.

Theorem 6.1 Let $a, b, m, n \geqslant 1$. Then

$$
\frac{1-q^{\operatorname{gcd}(a m, m+n)}}{1-q^{m+n}}\left[\begin{array}{c}
a m+b m-1 \\
a m
\end{array}\right]_{q}\left[\begin{array}{c}
a n+b n \\
a n
\end{array}\right]_{q}
$$

is a polynomial in $q$ with non-negative integer coefficients.

Corollary 6.2 Let $a, b, m, n \geqslant 1$. Then

$$
\frac{1-q^{a m}}{1-q^{m+n}}\left[\begin{array}{c}
a m+b m-1 \\
a m
\end{array}\right]_{q}\left[\begin{array}{c}
a n+b n \\
a n
\end{array}\right]_{q}
$$

is a polynomial in $q$ with non-negative integer coefficients.

It is easily seen that Theorem 1.4 can be obtained upon letting $q \rightarrow 1$ in Corollary 6.2. Moreover, when $a=b=m=1$, the numbers (6.2) reduce to the $q$-Catalan numbers

$$
C_{n}(q)=\frac{1-q}{1-q^{2 n+1}}\left[\begin{array}{c}
2 n \\
n
\end{array}\right]_{q}
$$


It is well known that the $q$-Catalan numbers $C_{n}(q)$ are polynomials with non-negative integer coefficients (see $[2,3,5,7]$ ). There are many different $q$-analogues of the Catalan numbers (see Fürlinger and Hofbauer [7]). For the so-called $q, t$-Catalan numbers, see $[8,13,12]$.

Recall that a polynomial $P(q)=\sum_{i=0}^{d} p_{i} q^{i}$ in $q$ of degree $d$ is called reciprocal if $p_{i}=p_{d-i}$ for all $i$, and that it is called unimodal if there is an integer $r$ with $0 \leqslant r \leqslant d$ and $0 \leqslant p_{0} \leqslant \cdots \leqslant p_{r} \geqslant \cdots \geqslant p_{d} \geqslant 0$. An elementary but crucial property of reciprocal and unimodal polynomials is the following.

Lemma 6.3 If $A(q)$ and $B(q)$ are reciprocal and unimodal polynomials, then so is their product $A(q) B(q)$.

Lemma 6.3 is well known and its proof can be found, e.g., in [1] or [16, Proposition 1].

Similarly to the proof of [11, Theorem 3.1], the following lemma plays an important role in the proof of Theorem 6.1. It is a slight generalization of [14, Proposition 10.1.(iii)], which extracts the essentials out of Andrews [4, Proof of Theorem 2].

Lemma 6.4 Let $P(q)$ be a reciprocal and unimodal polynomial and $m$ and $n$ positive integers with $m \leqslant n$. Furthermore, assume that $A(q)=\frac{1-q^{m}}{1-q^{n}} P(q)$ is a polynomial in $q$. Then $A(q)$ has non-negative coefficients.

Proof. See [11, Lemma 5.1].

Proof of Theorem 6.1. It is well known that the $q$-binomial coefficients are reciprocal and unimodal polynomials in $q$ (cf. [17, Ex. 7.75.d]), and by Lemma 6.3, so is the product of two $q$-binomial coefficients. In view of Lemma 6.4 , for proving Theorem 6.1 it is enough to show that the expression (6.1) is a polynomial in $q$. We shall accomplish this by a count of cyclotomic polynomials.

Recall the well-known fact that

$$
q^{n}-1=\prod_{d \mid n} \Phi_{d}(q)
$$

where $\Phi_{d}(q)$ denotes the $d$-th cyclotomic polynomial in $q$. Consequently,

$$
\frac{1-q^{\operatorname{gcd}(a m, m+n)}}{1-q^{m+n}}\left[\begin{array}{c}
a m+b m-1 \\
a m
\end{array}\right]_{q}\left[\begin{array}{c}
a n+b n \\
a n
\end{array}\right]_{q}=\prod_{d=2}^{\min \{a m+b m-1, a n+b n\}} \Phi_{d}(q)^{e_{d}},
$$

with

$$
\begin{aligned}
e_{d}= & \chi(d \mid \operatorname{gcd}(a m, m+n))-\chi(d \mid m+n)+\left\lfloor\frac{a m+b m-1}{d}\right\rfloor+\left\lfloor\frac{a n+b n}{d}\right\rfloor \\
& -\left\lfloor\frac{a m}{d}\right\rfloor-\left\lfloor\frac{b m-1}{d}\right\rfloor-\left\lfloor\frac{a n}{d}\right\rfloor-\left\lfloor\frac{b n}{d}\right\rfloor,
\end{aligned}
$$


where $\chi(\mathcal{S})=1$ if $\mathcal{S}$ is true and $\chi(\mathcal{S})=0$ otherwise. This is clearly non-negative, unless $d \mid m+n$ and $d \nmid \operatorname{gcd}(a m, m+n)$.

So, let us assume that $d \mid m+n$ and $d \nmid \operatorname{gcd}(a m, m+n)$, which means that $d \nmid a m$ and therefore

$$
\begin{aligned}
\left\lfloor\frac{a m+b m-1}{d}\right\rfloor+\left\lfloor\frac{a n+b n}{d}\right\rfloor & =\frac{(a+b)(m+n)}{d}-1, \\
\left\lfloor\frac{a m}{d}\right\rfloor+\left\lfloor\frac{a n}{d}\right\rfloor & =\frac{a(m+n)}{d}-1, \\
\left\lfloor\frac{b m-1}{d}\right\rfloor+\left\lfloor\frac{b n}{d}\right\rfloor & =\frac{b(m+n)}{d}-1,
\end{aligned}
$$

and so $e_{d}=0$ is also non-negative in this case. This completes the proof of polynomiality of (6.1).

Proof of Corollary 6.2. This follows immediately from Theorem 6.1 and the fact that $\operatorname{gcd}(a m, m+n) \mid a m$.

\section{Concluding remarks and open problems}

On January 2, 2014 T. Amdeberhan and V.H. Moll (personal communication) found the following generalization of Theorem 1.1, which was soon proved by Q.-H. Yang [21] and C. Krattenthaler.

Conjecture 7.1 Let $a, b$ and $n$ be positive integers with $a>b$. Then

$$
(2 b n+1)(2 b n+3)\left(\begin{array}{c}
2 b n \\
b n
\end{array}\right) \mid 3(a-b)(3 a-b)\left(\begin{array}{c}
2 a n \\
a n
\end{array}\right)\left(\begin{array}{c}
a n \\
b n
\end{array}\right) .
$$

Let $[m] !=(1-q) \cdots\left(1-q^{m}\right)$. By a result of Warnaar and Zudilin [20, Proposition 3], one sees that, for any positive integer $n$, the polynomial

$$
\frac{[6 n] ![n] !}{[3 n] ![2 n] !^{2}}
$$

has non-negative integer coefficients. Similarly as before, we can prove the following generalization of congruences (1.3)-(1.5).

Theorem 7.2 Let $n$ be a positive integer. Then all of

$$
\begin{aligned}
& \frac{(1-q)[6 n] ![n] !}{\left(1-q^{2 n+1}\right)[3 n] ![2 n] !^{2}}, \quad \frac{\left(1-q^{3}\right)[6 n] ![n] !}{\left(1-q^{2 n+3}\right)[3 n] ![2 n] !^{2}}, \quad \frac{(1-q)\left(1-q^{3}\right)[6 n] ![n] !}{\left(1-q^{2 n+1}\right)\left(1-q^{2 n+3}\right)[3 n] ![2 n] !^{2}}, \\
& \frac{\left(1-q^{3}\right)\left(1-q^{5}\right)\left(1-q^{7}\right)[6 n] ![n] !}{\left(1-q^{2 n+3}\right)\left(1-q^{2 n+5}\right)\left(1-q^{2 n+7}\right)[3 n] ![2 n] !^{2}} \quad(n \geqslant 2), \\
& \frac{\left(1-q^{3}\right)^{2}\left(1-q^{5}\right)\left(1-q^{7}\right)[6 n] ![n] !}{\left(1-q^{2 n+1}\right)\left(1-q^{2 n+3}\right)\left(1-q^{2 n+5}\right)\left(1-q^{2 n+7}\right)[3 n] ![2 n] !^{2}} \quad(n \geqslant 2)
\end{aligned}
$$

are polynomials in $q$. 
We have the following two related conjectures.

Conjecture 7.3 All the polynomials in Theorem 7.2 have non-negative integer coefficients.

Conjecture 7.4 Let $n \geqslant 2$. Then the polynomial $\frac{[6 n] ![n] !}{[3 n] ![2 n] !^{2}}$ is unimodal.

It is obvious that the polynomial $\frac{[6 n] ![n] !}{[3 n] ![2 n] ! 2}$ is reciprocal. If Conjecture 7.4 is true, then, applying Lemma 6.3, we conclude that the first two polynomials in Theorem 7.2 have non-negative integer coefficients.

It was conjectured by Warnaar and Zudilin (see [20, Conjecture 1]) that

$$
\frac{[15 n] ![2 n] !}{[10 n] ![4 n] ![3 n] !}
$$

has non-negative integer coefficients. Similarly, we have the following generalization of Theorem 1.2.

Theorem 7.5 Let $n$ be a positive integer. Then both

$$
\frac{(1-q)[15 n] ![2 n] !}{\left(1-q^{10 n+1}\right)[10 n] ![4 n] ![3 n] !}, \quad \text { and } \quad \frac{\left(1-q^{3}\right)\left(1-q^{7}\right)[15 n] ![2 n] !}{(1-q)\left(1-q^{10 n+3}\right)[10 n] ![4 n] ![3 n] !}
$$

are polynomials in $q$.

We end the paper with the following conjecture, strengthening the above theorem.

Conjecture 7.6 The two polynomials in Theorem 7.5 have non-negative integer coefficients.

Acknowledgments. The author thanks Quan-Hui Yang, T. Amdeberhan and V.H. Moll for helpful comments on a previous version of this paper. This work was partially supported by the Fundamental Research Funds for the Central Universities and the National Natural Science Foundation of China (grant 11371144).

\section{References}

[1] G.E. Andrews, A theorem on reciprocal polynomials with applications to permutations and compositions, Amer. Math. Monthly 82 (1975), 830-833.

[2] G.E. Andrews, Catalan numbers, $q$-Catalan numbers and hypergeometric series, $J$. Combin. Theory Ser. A 44 (1987), 267-273.

[3] G.E. Andrews, On the difference of successive Gaussian polynomials, J. Statist. Plann. Inference 34 (1993), 19-22. 
[4] G.E. Andrews, The Friedman-Joichi-Stanton monotonicity conjecture at primes, Unusual Applications of Number Theory (M. Nathanson, ed.), DIMACS Ser. Discrete Math. Theor. Comp. Sci., vol. 64, Amer. Math. Soc., Providence, R.I., 2004, pp. 9-15.

[5] G.E. Andrews, q-Catalan identities, in: The Legacy of Alladi Ramakrishnan in the Mathematical Sciences, Springer, New York, 2010, pp. 183-190.

[6] J.W. Bober, Factorial ratios, hypergeometric series, and a family of step functions, J. Lond. Math. Soc. 79 (2009), 422-444.

[7] J. Fürlinger and J. Hofbauer, q-Catalan numbers, J. Combin. Theory Ser. A 40 (1985), 248-264.

[8] A.M. Garsia and J. Haglund, A proof of the $q, t$-Catalan positivity conjecture, Discrete Math. 256 (2002), 677-717.

[9] I.M. Gessel, Super ballot numbers, J. Symbolic Comput. 14 (1992), 179-194.

[10] V.J.W. Guo, Proof of Sun's conjecture on the divisibility of certain binomial sums, Electron. J. Combin. 20(4) (2013), \#P20.

[11] V.J.W. Guo and C. Krattenthaler, Some divisibility properties of binomial and qbinomial coefficients, J. Number Theory 135 (2014), 167-184.

[12] J. Haglund, Conjectured statistics for the q,t-Catalan numbers, Adv. Math. 175 (2003), 319-334.

[13] M. Haiman, t, q-Catalan numbers and the Hilbert scheme, Discrete Math. 193 (1998), 201-224.

[14] V. Reiner, D. Stanton, and D. White, The cyclic sieving phenomenon, J. Combin. Theory Ser. A 108 (2004), 17-50.

[15] M.R. Sepanski, On divisibility of convolutions of central binomial coefficients, Electron. J. Combin. 21(1) (2014), \#P1.32.

[16] R.P. Stanley, Log-concave and unimodal sequences in algebra, combinatorics, and geometry, in: Graph Theory and Its Applications: East and West (Jinan, 1986), Ann. New York Acad. Sci., 576, New York Acad. Sci., New York, 1989, pp. 500-535.

[17] R.P. Stanley, Enumerative Combinatorics, vol. 2, Cambridge University Press, Cambridge, 1999.

[18] Z.-W. Sun, On divisibility of binomial coefficients, J. Austral. Math. Soc. 93 (2012), 189-201.

[19] Z.-W. Sun, Products and sums divisible by central binomial coefficients, Electron. J. Combin. 20(1) (2013), \#P9.

[20] S. O. Warnaar and W. Zudilin, A q-rious positivity, Aequat. Math. 81 (2011), $177-$ 183.

[21] Q.-H. Yang, Proof of a conjecture related to divisibility properties of binomial coefficients, preprint, 2014, arXiv:1401.1108. 